\title{
Patients présentant un syndrome coronarien aigu avec sus-décalage du segment ST admis en structure d'urgence après régulation par un Centre 15
}

\section{Patients with ST-Elevation Myocardial Infarction Admitted in Emergency Department after Medical Regulation}

\section{Cachera $\cdot$ F. Balen $\cdot$ S. Charpentier}

Reçu le 19 décembre 2018; accepté le 26 avril 2019

(C) SFMU et Lavoisier SAS 2019

Résumé Introduction : La cardiopathie ischémique est une des principales causes de mortalité en Europe. Cette mortalité importante est l'apanage particulier des syndromes coronariens avec élévation du segment ST (SCA ST+). Si la prise en charge préhospitalière de ces patients (de l'appel à la table de coronarographie) est sous la responsabilité des Samu, certains patients échappent à la filière malgré un appel au Centre 15. Notre objectif était de décrire ces patients porteurs d'un SCA ST+, admis en structure d'urgence (SU) après régulation par un Centre 15.

Méthode : Il s'agit d'une étude observationnelle de cohorte rétrospective incluant tous les patients admis en $\mathrm{SU}$ du CHU de Toulouse du $1^{\text {er }}$ avril 2015 au 31 janvier 2017 avec un diagnostic final de SCA ST+.

Résultats : Sur les 139 patients qui ont été admis en SU pour SCA ST+, 96 (69\%) avaient contacté le Centre 15. Les dossiers de 89 patients ont été analysés. On retrouve une importante proportion de patientes de plus de 75 ans, qui représentent un quart de la population. Seulement 30 patients (34\%) présentaient une douleur typique.

Discussion : Notre étude retrouve un taux de douleurs atypiques et une proportion de femmes âgées plus importante que la littérature portant sur la description des patients porteurs de SCA ST+. Ces atypies de présentation peuvent expliquer la difficulté à réguler et orienter ces patients.

Mots clés $\mathrm{SCA} \mathrm{ST}+\cdot$ Structures d'urgence

D. Cachera $(\bowtie) \cdot$ F. Balen $\cdot$ S. Charpentier

Pole de médecine d'urgence/Samu,

CHU de Toulouse, place du Docteur-Baylac,

TSA 40031, F-31059 Toulouse cedex 09, France

e-mail : cachera.d@chu-toulouse.fr

F. Balen $\cdot$ S. Charpentier

Université Toulouse-III-Paul-Sabatier,

route de Narbonne, F-31330 Toulouse, France
Abstract Introduction: Ischemic heart disease is one of the leading causes of death in Europe. ST-elevation myocardial infarction is the deadliest event of the ischemic heart disease. In France, the optimal pathway for those patients is to call the medical dispatch center (MDC) that should recognize the STEMI patient calling for chest pain and send patient directly to the Cath lab. The objective of our study was to describe patients admitted in the emergency department (ED) instead of Cath lab after having called the MDC.

Method: This is an observational and retrospective cohort study on STEMI patients admitted to the ED of the University Hospital of Toulouse from April 1, 2015 to January 31, 2017.

Results: One hundred thirty-nine patients were admitted to the ED for STEMI. Ninety-six (69\%) patients had called the MDC. Eighty-nine patients were analyzed. About $25 \%$ of them were women older than 75 years old. Only 30 patients (34\%) analyzed had typical chest pain.

Discussion: Our study shows an important proportion of atypical chest pain and of old women, compared to the STEMI population usually described in the literature. This could explain the difficulty to evaluate the probability of STEMI when a patient calls MDC for chest pain.

Keywords STEMI $\cdot$ Emergency Department

\section{Introduction}

La cardiopathie ischémique est une des principales causes de mortalité en Europe [1]. En France, l'incidence des syndromes coronariens aigus (SCA) est estimée à 2500 par million d'habitants [2]. Avant les années 2000, la mortalité de l'infarctus du myocarde (IDM) [incluant, selon les définitions, les SCA ST+ et non ST+] était de $50 \%$ à un mois, dont $25 \%$ 
dans les deux premières heures, en l'absence de prise en charge [3]. Cette mortalité importante est l'apanage particulier des SCA avec élévation du segment ST (SCA ST+), correspondant à une occlusion complète de l'artère coronaire. Les progrès thérapeutiques et l'organisation des filières de soins ont permis de réduire cette mortalité intrahospitalière à $5 \%$ dans les années 2005 [4] puis de 2,8\% dans les années 2015 [5]. La filière de soin du SCA recommandée par l'ESC [6-7] englobe la prise en charge préhospitalière incluant la thrombolyse sur site ou le transfert direct sur table d'angioplastie et a pour objectif de réduire le délai de reperfusion coronaire. La stratégie française de prise en charge des SCA ST+ est fondée sur l'utilisation des Samu-Smur [8]. Un patient présentant une douleur thoracique est incité à contacter le Centre 15 où la probabilité de SCA ST+ est évaluée par un médecin régulateur. Un patient présentant un SCA ST+ doit bénéficier du déclenchement d'une équipe Smur, ce qui permet de réduire le délai d'accès à une reperfusion. Un patient présentant un SCA ST+ ayant contacté la régulation du Centre 15 ne devrait donc pas être adressé en structure d'urgence (SU), mais directement en coronarographie par transport médicalisé, ou directement en unité de soins intensifs de cardiologie (USIC) après thrombolyse. Nonobstant, en 2008-2010, dans notre région, $20 \%$ des patients présentant un SCA ST+ ont été admis par les SU [9]. Cela peut être du fait que le patient se présente directement en SU, mais également d'une mauvaise évaluation lors de la régulation médicale, liée à la difficulté d'évaluer au téléphone la probabilité de SCA chez un patient appelant le Centre 15 pour douleur thoracique.

L'objectif principal de notre étude est de décrire la population des patients porteurs de SCA ST+ adressés en SU après régulation médicale par un Centre 15 .

\section{Méthode}

Il s'agit d'une étude observationnelle de cohorte rétrospective monocentrique menée sur le CHU de Toulouse. Ce recueil a été réalisé dans le cadre du registre RESCAMIP (Registre des SCA ST+ de Midi-Pyrénées mené entre mars 2015 et mars 2017) ayant fait l'objet d'une déclaration à la CNIL (numéro d'autorisation 914329).

Tous les patients admis en SU du CHU de Toulouse du $1^{\text {er }}$ avril 2015 au 31 janvier 2017 avec diagnostic final de SCA ST+ étaient éligibles. Les patients adressés par le médecin généraliste ou venus spontanément ont été exclus. Les dossiers incomplets ont également été exclus.

Les caractéristiques démographiques (âge, sexe, facteurs de risque et antécédents cardiovasculaires), anamnestiques (symptomatologie, typologie de la douleur, délai d'appel au Centre 15, délai de transport en SU) et cliniques (paramètres vitaux) ont été relevées sur dossiers de régulation à l'aide du logiciel AppliSAMU. Une douleur thoracique était décrite comme typique si rétrosternale ou thoracique gauche, constrictive, en barre ou en étau, avec ou sans irradiation dans le bras gauche. En l'absence de description, la douleur était considérée comme atypique.

\section{Analyse statistique}

L'analyse statistique des données anonymisées a été réalisée à l'aide du logiciel ExcelC (version 15.20, 2016 Microsoft, Redmond, Washington, Etats-Unis). Les valeurs manquantes ont été considérées comme absentes. La distribution des variables quantitatives a été représentée par la moyenne suivie de l'écart-type si leur distribution était normale, par la médiane suivie du premier quartile (p25\%) et du troisième quartile de distribution (p75 \%) si non. Les données qualitatives ont été exprimées en nombre et en pourcentage.

\section{Résultats}

Sur la période analysée de 23 mois, 139 patients ont été admis en SU du CHU de Toulouse. Parmi eux, 7 (5 \%) étaient adressés par leur médecin traitant (MT) et 96 (69 \%) avaient contacté le Centre 15. Sept dossiers manquants ont été exclus, l'analyse porte donc sur 89 patients. Le diagramme de flux est représenté sur la figure 1.

Les caractéristiques des patients admis pour SCA ST+ en SU après régulation médicale sont représentées dans le tableau 1. La population avait une moyenne d'âge de 69 ans et était en majorité masculine (48 hommes [54\%]). À noter que 22 patients, soit $25 \%$ de la population totale était représentée par des femmes de plus de 75 ans. La répartition de la population par tranche d'âge et par sexe est présentée sur la figure 2. Il existait une notion de cardiopathie ischémique chez 14 patients $(16 \%)$ et d'accident vasculaire cérébral (AVC) chez six (7\%) autres patients. Hormis l'hypertension artérielle (HTA), qui était présente chez 36 sujets (40\%), peu de facteurs de risque avaient été relevés en régulation. La douleur thoracique était décrite comme typique chez seulement 30 sujets (34\%). Il faut cependant noter un nombre élevé de douleurs non décrites dans le dossier de régulation médicale $(n=35$ [39\%]). Le délai entre l'apparition des symptômes et l'appel à la régulation avait une médiane à 85 minutes (interquartile $=18-281$ ). Le délai entre l'appel à la régulation et l'arrivée aux urgences avait une médiane à 79 minutes (interquartile $=68-104$ ).

\section{Discussion}

Parmi les patients porteurs de SCA ST+ admis dans nos SU sur la période d'inclusion, la majorité avait été régulée. 


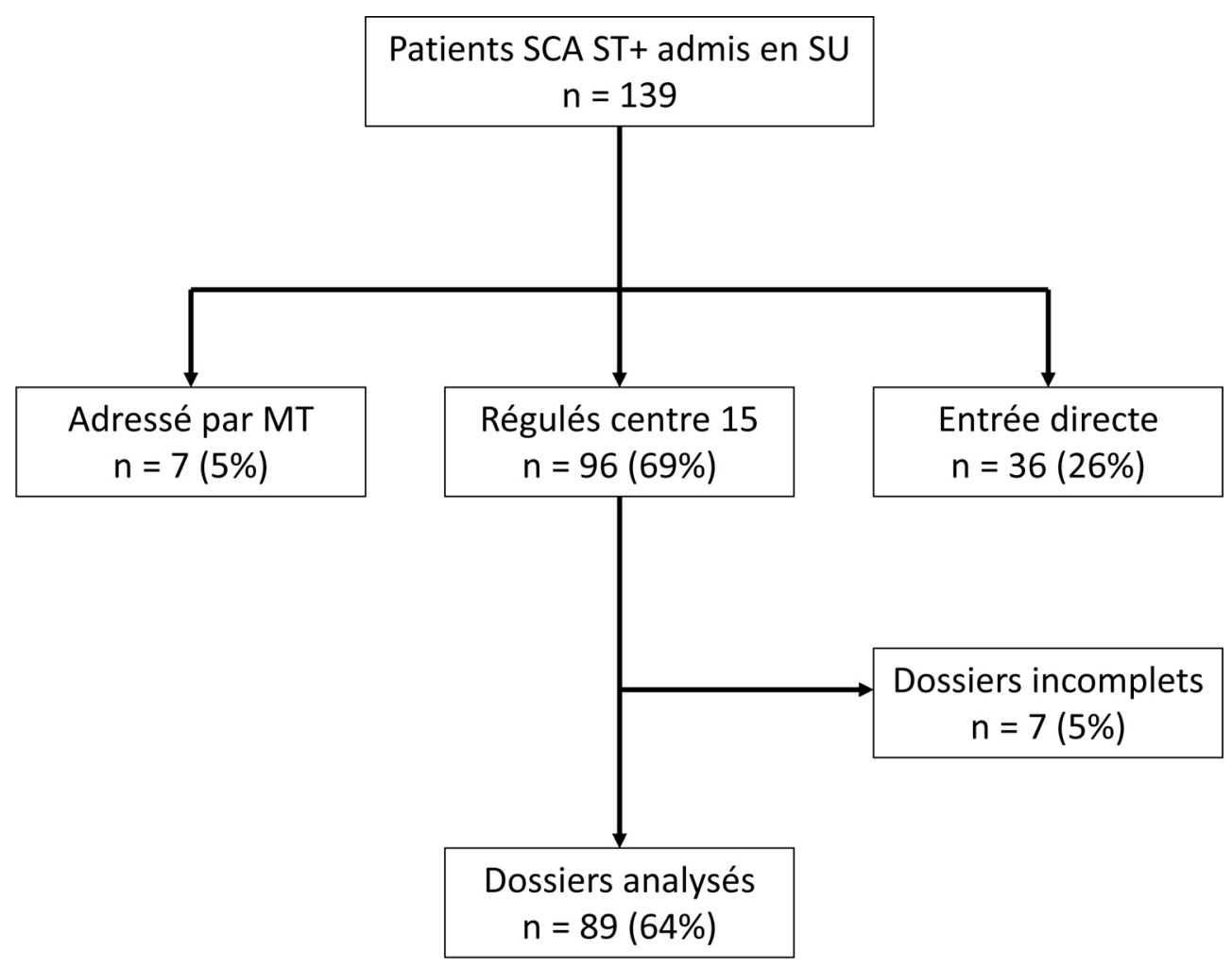

Fig. 1 Diagramme de flux SCA ST+ : syndrome coronarien aigu avec élévation du segment ST ; MT : médecin traitant

\begin{tabular}{|ll|}
\hline \multicolumn{2}{|l|}{ Tableau 1 Caractéristiques de la population } \\
\hline & Population $(\mathbf{n}=\mathbf{8 9})$ \\
\hline Âge (ans) & $69 \pm 19$ \\
Sexe féminin & $41(46 \%)$ \\
Antécédents cardiovasculaires & \\
Cardiopathie ischémique & $14(16 \%)$ \\
Accident vasculaire cérébral & $6(7 \%)$ \\
Facteurs de risque cardiovasculaires & \\
Hypertension artérielle & $36(40 \%)$ \\
Tabagisme & $17(19 \%)$ \\
Dyslipidémie & $11(12 \%)$ \\
Diabète & $10(11 \%)$ \\
Antécédents familiaux & $8(9 \%)$ \\
Typologie de la douleur thoracique & \\
Typique & $30(34 \%)$ \\
Atypique & $24(27 \%)$ \\
Non décrite & $35(39 \%)$ \\
Stratégie de reperfusion & $80(90 \%)$ \\
Angioplastie primaire & $3(3 \%)$ \\
Thrombolyse & $6(7 \%)$ \\
Aucune & \\
\hline Données exprimées en nombre $(\%)$ & \\
\hline
\end{tabular}

Il s'agit d'une population présentant peu de facteurs de risque et une symptomatologie atypique. On note une part importante de femmes de plus de 75 ans.

Les principales limites de l'étude tiennent à son caractère rétrospectif et monocentrique, responsable du faible effectif et du nombre de données manquantes. Cependant, nous pouvons assurer l'exhaustivité des patients inclus sur la période, et il était important de décrire le profil de ces patients « oubliés de la filière » des SCA ST+ malgré leur appel au Centre 15. À noter que le registre RESCAMIP sur la période de mars 2015 à mars 2017 a enregistré 666 patients porteurs de SCA ST+ en Haute-Garonne [10].

Concernant le caractère atypique de la douleur des patients présentant un SCA ST+, nos résultats sont cohérents avec la littérature. En effet, $15 \%$ de l'ensemble des patients porteurs de SCA ST+ présenteraient une douleur atypique [5]. Si on ne s'intéresse qu'aux patients appelant le Centre 15, la proportion de douleurs atypiques parmi les SCA $\mathrm{ST}+$ serait proche de $40 \%$ [11]. Dans notre cohorte de patients adressés en SU après régulation médicale, le taux de douleur typique est faible (34\%). Le caractère atypique de la douleur chez ces patients peut expliquer la difficulté de leur orientation en régulation médicale, car ils ne bénéficient alors souvent que de l'envoi de pompiers ou d'ambulanciers privés, ne disposant pas à l'heure actuelle d'ECG télétransmis. 
25

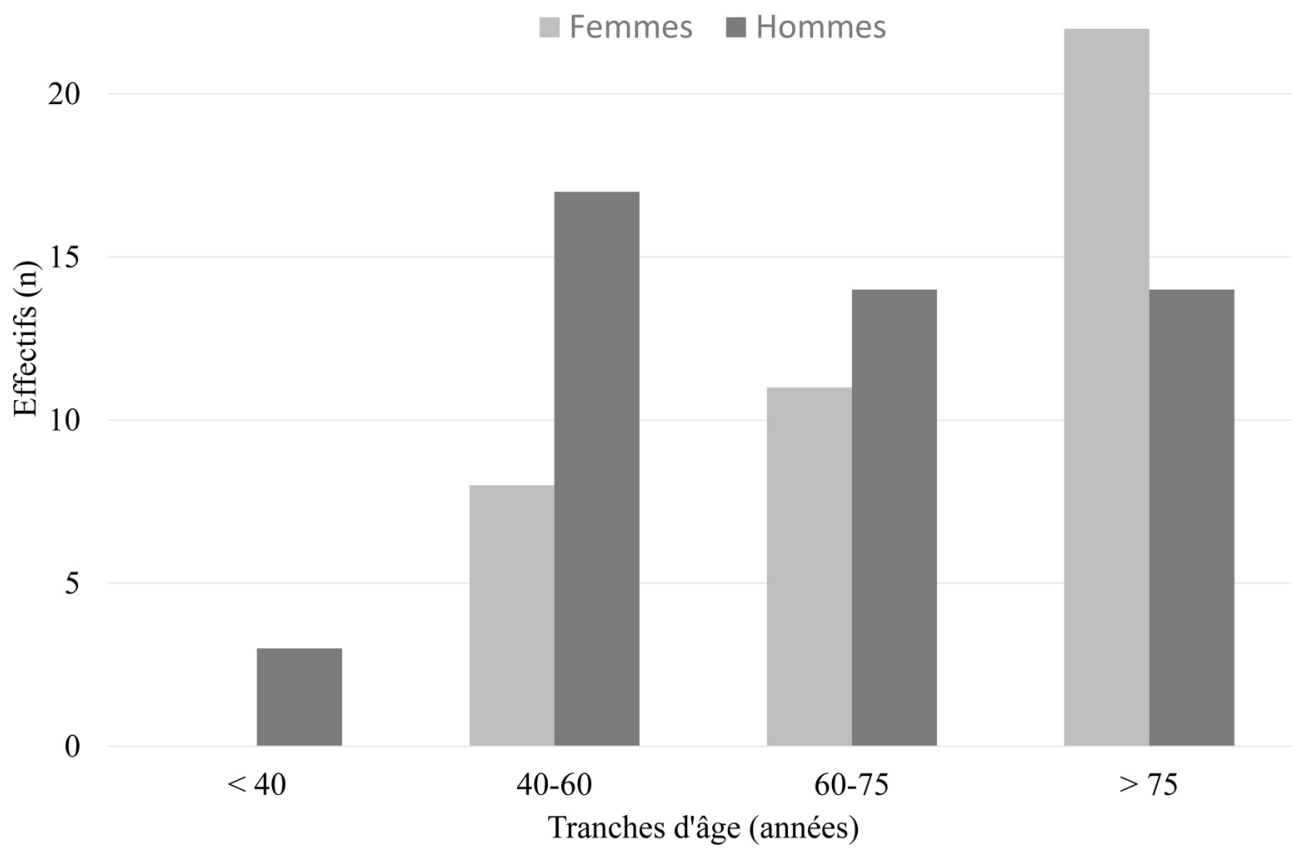

Fig. 2 Répartition des patients par sexe et tranche d'âge

Il est important de noter la proportion de femmes de $46 \%$ dans notre cohorte, alors qu'elles sont habituellement moins représentées dans les cohortes de patients atteints de SCA ST +. C'est le cas dans le travail de Manzo-Silberman et al. [12] ou les femmes ne représentent que $24 \%$ de la population des $\mathrm{SCA} \mathrm{ST}+$, mais ont une mortalité intrahospitalière trois fois plus importante. Cette surmortalité retrouvée dans la littérature semble expliquée par le délai de prise en charge des patientes présentant un SCA ST+ qui ont, comme dans notre cohorte, une symptomatologie atypique $[13,14]$ retardant le diagnostic [15].

\section{Conclusion}

Les patients porteurs de $\mathrm{SCA} \mathrm{ST}+$ admis aux urgences après régulation médicale par un Centre 15 présentent une symptomatologie atypique. On note une proportion importante parmi eux de femmes âgées de plus de 75 ans.

Liens d'intérêts : les auteurs déclarent ne pas avoir de liens d'intérets.

\section{Références}

1. Townsend N, Wilson L, Bhatnagar P, et al (2016) Cardiovascular disease in Europe: epidemiological update 2016. Eur Heart J $37: 3232-45$
2. Danchin N, Puymirat E, Aissaoui N, et al (2010) Épidémiologie des syndromes coronaires aigus en France et en Europe. Ann Cardiol Angeiol 59:37-41

3. Tunstall-Pedoe H, Kuulasmaa K, Mähönen M, et al (1999) Contribution of trends in survival and coronary-event rates to changes in coronary heart disease mortality: 10-year results from 37 WHO MONICA Project populations. Lancet 353:1547-57

4. Cambou J-P, Simon T, Mulak G, et al (2007) The French registry of Acute ST elevation or non-ST-elevation Myocardial Infarction (FAST-MI): study design and baseline characteristics. Arch Mal Coeur Vaiss 100:524-34

5. Belle L, Cayla G, Cottin Y, et al (2017) French Registry on Acute ST-elevation and non-ST-elevation Myocardial Infarction 2015 (FAST-MI 2015). Design and baseline data. Arch Mal Coeur Vaiss 110:366-78

6. Windecker S, Kolh P, Alfonso F, et al (2014) ESC/EACTS Guidelines on myocardial revascularization: the Task Force on myocardial revascularization of the European Society of Cardiology (ESC) and the European Association for Cardio-Thoracic Surgery (EACTS) Developed with the special contribution of the European Association of Percutaneous Cardiovascular Interventions (EAPCI). Eur Heart J 35:2541-619

7. Ibanez B, James S, Agewall S, et al (2018) ESC Guidelines for the management of acute myocardial infarction in patients presenting with ST-segment elevation: the Task Force for the management of acute myocardial infarction in patients presenting with ST-segment elevation of the European Society of Cardiology (ESC). Eur Heart J 39:119-77

8. Haute Autorité de santé (2012) Recommandations professionnelles sur la prise en charge de l'infarctus du myocarde à la phase aiguë en dehors des services de cardiologie. https://www.hassante.fr/portail/upload/docs/application/pdf/2012-11/05c01_conf_ consensus_infarctus_myocarde_recos_courtes.pdf (Dernier accès le 15 avril 2019 ) 
9. Sandouk A, Ducassé JL, Grolleau S, et al (2012) Compliance with guidelines in patients with ST-segment elevation myocardial infarction after implementation of specific guidelines for emergency care: results of RESCA +31 registry. Arch Mal Coeur Vaiss 105:262-70

10. Balen F, Lhermusier T, Grolleau S, et al (2019) Identifying key factors leading to the optimal care pathway for patients with STsegment elevation myocardial infarction: results from the RESCAMIP registry. Arch Mal Coeur Vaiss (sous presse)

11. Charpentier S, Beaune S, Joly LM, et al (2017) Management of chest pain in the French emergency healthcare system: the prospective observational EPIDOULTHO study. Eur J Emerg Med 25:404-10

12. Manzo-Silberman S, Couturaud F, Charpentier S, et al (2017) Influence of gender on delays and early mortality in ST-segment elevation myocardial infarction: insight from the first French Metaregistry, 2005-2012 patient-level pooled analysis. Int J Cardiol 262:1-8

13. Mnatzaganian G, Braitberg G, Hiller JE, et al (2016) Sex differences in in-hospital mortality following a first acute myocardial infarction: symptomatology, delayed presentation, and hospital setting. BMC Cardiovasc Disord 16:109

14. Goldberg RJ, O’Donnell C, Yarzebski J, et al (1998) Sex differences in symptom presentation associated with acute myocardial infarction: a population-based perspective. Am Heart J 136:189-95

15. Meischke H, Larsen MP, Eisenberg MS (1998) Gender differences in reported symptoms for acute myocardial infarction: impact on prehospital delay time interval. Am J Emerg Med 16:363-6 